\title{
Aprendizagem auto-regulada: Fundamentos e organização do Programa SABER
}

\author{
Programa de auto-regulação da aprendizagem
}

\author{
Helena Bilimória \\ Leandro S. Almeida
}

\begin{abstract}
Resumo
Este artigo apresenta um programa de promoção da auto-regulação da aprendizagem escolar para adolescentes. À luz das teorias da auto-regulação, em particular do modelo do adaptable learning, as 12 sessões do programa desenvolvem competências de análise e de definição de objectivos, de planeamento e de avaliação das tarefas de aprendizagem e de resolução de problemas. Neste quadro, as actividades do programa cobrem componentes motivacionais, cognitivas e metacognitivas da aprendizagem. Por sua vez, à luz das teorias construtivistas da aprendizagem aposta-se numa dinâmica de treino assente, em primeiro lugar, na realização individual das tarefas, seguida da discussão em grupo das estratégias usadas e resultados atingidos. A par da estrutura organizativa das 12 sessões do programa, descreve-se a estrutura interna de cada sessão, destacando-se a dinâmica das mesmas e os procedimentos seguidos de forma a maximizar o envolvimento e a auto-regulação dos alunos.
\end{abstract}

Palavras-chave: estratégias de aprendizagem; auto-regulação da aprendizagem; metacognição.

\section{Self-regulated learning: theoretical foundations and organization of SABER program}

\begin{abstract}
This paper presents a self-regulated learning training program for adolescents. On basis of self-regulated, namely adaptable learning model, the twelve sessions program promote the analyses and goals definition, as well as planning and evaluation competencies on learning and problem solving tasks. Program activities include motivation, cognition and metacognition learning components. We also consider the constructivist approaches of learning in order to organize the training dynamic. First, we assume an individual execution of the activities, then we promote a group discussion concerning the strategies implemented and the results obtained. We describe the structure of the twelve sessions and the internal structure of each session, particularly their dynamic and procedures to maximize the students' engagement and self-regulation development.
\end{abstract}

Keywords: learning strategies; self regulated learning; metacognition.

\section{Aprendizaje auto-regulada: Fundamentos y organización del Programa SABER}

\section{Resumen}

Este artículo presenta un programa de promoción de la auto-regulación del aprendizaje escolar para adolescentes. Con base en las teorías de la autoregulación, particularmente del modelo adaptable learning, las 12 sesiones del programa desarrollan competencias de análisis y de definición de objetivos, de planificación y de evaluación de las tareas de aprendizaje y de resolución de problemas. En ese sentido las actividades del programa abarcan componentes de motivación, cognitivos y meta cognitivos del aprendizaje. Por su vez, con base en las teorías constructivistas del aprendizaje se apuesta en una dinámica de entrenamiento permanente, en primer lugar, en la realización individual de las tareas, seguido de la discusión en grupo de las estrategias utilizadas y los resultados alcanzados. En la estructura organizativa de las 12 sesiones del programa se describe la estructura interna de cada sesión, destacándose su dinámica y los procedimientos utilizados, como una forma de maximizar el envolvimiento y la auto-regulación de los alumnos.

Palabras clave: estrategias de aprendizaje; auto-regulación del aprendizaje; meta cognición. 


\section{Introdução}

$\mathrm{Na}$ actualidade, o construtivismo e o coconstrutivismo constituem-se como os dois pilares essenciais de qualquer teoria relativa ao processo de aprendizagem. Aliás, o próprio processo de aprendizagem, e desde o século transacto, deixou de ser encarado como um processo individual, passando a ser encarado como uma dimensão de um processo interactivo de co-construção que designamos, no seio da escola, por ensinoaprendizagem. Quer isto dizer, que a linguagem, a comunicação e a interacção social se tornaram no principal factor de aprendizagem enquanto construção e transformação progressiva de conhecimento. Aliás, para alguns autores (Doise \& Mugny, 1979, 1997; Vygotsky, 1978), tais variáveis são, não só decisivas para a aprendizagem, como também para o desenvolvimento cognitivo do indivíduo.

Efectivamente, e para Vygotsky (2003), a aprendizagem "é um aspecto necessário e universal do processo de desenvolvimento das funções psicológicas culturalmente organizadas e especificamente humanas" (p.118), sendo que o "processo de desenvolvimento segue de forma mais lenta e atrás do processo de aprendizagem" (p.118). O mesmo autor salienta o papel da linguagem na construção do pensamento, por meio do desenvolvimento da função planeadora que esta capacidade humana adquire. Refere assim Vygotsky (2003) que a linguagem, para além da função emotiva e comunicativa, apresenta também uma função planeadora, "habilitando as crianças a providenciarem instrumentos auxiliares na solução de tarefas difíceis, a superar a acção impulsiva, a planear a solução para um problema antes da sua execução e controlar o próprio comportamento" (p.38).

Descrevendo-se operativamente a interacção social como partilha, cooperação e confronto de informação, conhecimentos e posicionamentos, facilmente percebemos o seu efeito dinamizador nos indivíduos, facilitando a representação mental das tarefas, bem como repercutindo-se no controlo das actividades e nas actividades metacognitivas (Roux, 2003). Veiculado por meio do pensamento e da linguagem, esta interacção promove internalização, ou seja, uma conversão da fala, como função comunicativa, em fala interior. Assim, é após este processo de internalização que a linguagem passa a organizar o pensamento da criança, tornando-se uma função mental interna (Vygotsky, 2003, p.117). A conversação tem, pois, a propriedade de se constituir como uma matriz primária do cumprimento das relações sociais e do próprio pensamento, por meio do uso da linguagem (Trognon, 1999).

Também Doise e Mugny (1997) reportam-se à noção de conflito sócio-cognitivo salientando que a dinâmica do desenvolvimento cognitivo resulta de um "conflito de comunicação social" (p.53). Ou seja, é pela interacção social que diferentes centrações individuais dos participantes entram em conflito, gerando perturbação cognitiva mais directa na criança do que se o confronto apenas se operasse com objectos físicos. Mais especificamente, em certas fases do desenvolvimento, a acção comum de vários indivíduos com vista à resolução de um conflito de centrações resulta na construção de novas coordenações nos indivíduos, quer nos que 
estão menos desenvolvidos, quer nos que estão mais avançados em termos desenvolvimentais (PerretClermont, 1995).

\section{Complementarmente, Feuerstein} apresenta-nos a noção de mediatização, ou experiência mediatizada como forma de promover o desenvolvimento cognitivo, reiterando o papel do "Outro" na abordagem aos objectos/problemas e, consequentemente, no desenvolvimento cognitivo. $\mathrm{Na}$ experiência de aprendizagem mediatizada, ocorre uma interacção plena de intencionalidade na qual o mediatizador (educador) se situa entre o mediatizado e os estímulos de modo a seleccionálos, alterá-los, ampliá-los ou interpretá-los, usando estratégias interactivas que permitam a atribuição de significado e a transferência para outras situações (Fonseca, 2001). A mediatização pode então entender-se como um fenómeno psico-sóciocultural através do qual decorre toda a aquisição, estruturação e desenvolvimento das condutas superiores (Fonseca, 2001). Deste modo, pode-se afirmar que a interacção social é o cerne do desenvolvimento cognitivo através da passagem de uma regulação externa para uma regulação interna das funções cognitivas. Quer isto dizer, que se pode considerar que o desenvolvimento cognitivo se processa através de uma internalização de uma regulação externa, tendendo a linguagem a ser o instrumento simbólico e, acima de tudo, cultural que melhor permite e promove o desenvolvimento de outras funções cognitivas, por exemplo a metacognição e a auto-regulação cognitiva.

No que se refere à auto-regulação, este mecanismo consta de um processo cíclico de três fases: planeamento, controlo volitivo e do desempenho, e auto-reflexão. $\mathrm{Na}$ fase de planeamento assumem primordial importância, por um lado, a análise da tarefa, designadamente o delineamento de objectivos e a selecção de estratégias; por outro, as crenças automotivacionais, entre elas, a auto-eficácia. Já na fase de controlo volitivo e do desempenho, dois processos tornam-se essenciais: o auto-controlo e a auto-monitorização. $\mathrm{O}$ auto-controlo inclui as autoinstruções, as imagens mentais e a focalização da atenção; a auto-monitorização, envolve dois processos: o auto-registo e a auto-experimentação. Por fim, a fase de auto-reflexão, a qual irá surtir efeitos numa fase de planeamento ulterior, em particular nas expectativas de eficácia pessoal e de realização e, consequentemente, no estabelecimento de objectivos. Na fase de auto-reflexão existem dois processos principais: o auto-julgamento, envolvendo as atribuições causais e a autoavaliação mediante critérios, e as auto-reacções, envolvendo em particular a auto-satisfação e as inferências adaptativas (Zimmerman, 2000). Em síntese, a auto-regulação da aprendizagem, segundo Zimmerman, envolve os motivos, os objectivos e a percepção de eficácia pessoal, o método e estratégias, o planeamento e gestão do tempo, a realização monitorizada pelo pensamento e controlo da acção e da volição, ou a organização e estruturação do ambiente (Zimmerman \& Risemberg, 1997).

Especificamente no que concerne à autoregulação da aprendizagem, Boekaerts (1992, 1996) apresentou o modelo do Adaptable Learning, no qual salientava que o comportamento dos indivíduos era orientado por duas prioridades: aumentar os seus conhecimentos e competências, ou seja, os seus recursos, mas por outro lado, 
prevenir a perda ou dano do seu próprio bem-estar. De acordo com este modelo, qualquer situação de aprendizagem despoleta um processo de trocas entre a avaliação que é feita da situação e um modelo dinâmico interno de trabalho que se baseia na informação recebida de três fontes: o self - as expectativas de eficácia pessoal, a hierarquia de objectivos, os valores e crenças motivacionais; a tarefa em contexto - que inclui a interpretação da tarefa em si, das instruções e do contexto físico e social da mesma; e, por fim, o conhecimento e as competências que o indivíduo detém, incluindo o conhecimento declarativo e procedimental, o conhecimento estratégico e, ainda, metacognitivo, relevantes para a situação. A avaliação que é feita da situação de aprendizagem é única para cada situação e orienta o estabelecimento de objectivos (intenções de aprendizagem ou de coping), assim como a manutenção e procura de concretização dos mesmos (realização de actividades de acordo com o modo de mestria ou com o modo de coping) (Boekaerts \& Niemivirta, 2000). Deste modo, o significado (interpretação) e a avaliação atribuídas pelos alunos às situações de aprendizagem estarão na base dos tipos de objectivos estabelecidos e no modo seleccionado para os alcançar.

Por tudo isto, cada vez mais a escola começa a compreender que depende de si, não a transmissão de conteúdos, mas o desenvolvimento nos alunos de estratégias auto-reguladoras da aprendizagem. Isto é, cabe à escola a promoção de estratégias de aprendizagem, que tornem os alunos: intencionais, isto é, que dirigem a sua acção para um objectivo pré-autodeterminado; sensíveis às variáveis do contexto de ensino-aprendizagem; e, conscientes, exercendo controlo e monitorização metacognitiva sistematicamente (Almeida, 1993; Boekaerts, 1992, 1996; Monereo, Pozo \& Castelló, 2001; Rosário, 2004; Veiga Simão, 2002). Nesta linha, parece-nos relevante diferenciar estratégias de estudo (procedimentos intencionais e conscientes dirigidos a um objectivo de aprendizagem) e técnicas de estudo (procedimentos usados de forma mecânica, sem que haja intuito de desenvolvimento pessoal e autonomia reflexiva na forma de aprender).

Assim, pode-se afirmar que actualmente se defende uma aprendizagem menos receptiva e progressivamente mais baseada na construção autónoma, passando pela descoberta guiada (Novak \& Gowin, 1999). Independentemente do modo como a aprendizagem se processa, o mais relevante é a que esta seja significativa, ou seja, que um novo conceito se relaciona com um outro conceito já presente na estrutura cognitiva do indivíduo. $\mathrm{O}$ novo conceito pode ser, deste modo, assimilado por um conceito subordinador, mais inclusivo, daqui resultando uma aprendizagem subordinada; ou um conceito mais inclusivo pode ser assimilado a partir de conceitos menos abrangentes, com ele relacionados, presentes na estrutura cognitiva do indivíduo, produzindo uma aprendizagem superordenada (Ausubel, 1968; Moreira \& Buchweitz, 2000). De qualquer modo, uma intervenção que vise melhorar a aprendizagem tem que atribuir ao estudante o protagonismo na acção, dado que é a sua actividade de selecção, filtragem, codificação, organização e processamento da informação que o conduz a construir os conteúdos da aprendizagem e a desenvolver as competências associadas (González-Pienda, 2003).

É neste contexto de uma progressiva responsabilização da escola pelas estratégias de 
aprendizagem que os seus alunos usam, ou não usam, assim como de reconhecimento do papel central do aluno, como um ser activo, dinâmico e auto-regulado, na construção do seu conhecimento, que desenvolvemos o programa SABER (saber aprender boas estratégias de regulação). Este programa integra-se no movimento do ensinar a pensar, tomando o aprender a aprender como a base essencial para uma auto-regulação das aprendizagens e consequente sucesso escolar (Almeida, 1993; Rosário, 2004). O programa fundamenta-se, assim, nas teorias construtivistas e co-construtivistas da aprendizagem, e do funcionamento cognitivo, bem como nos modelos de aprendizagem auto-regulada, em particular, no modelo do adaptable learning (Boekaerts, 1992, 1996). O seu objectivo primeiro é promover a autoregulação da aprendizagem nos alunos, através da internalização de estratégias de aprendizagem diversas, contextualizadas e avaliadas nos seus custos e efeitos. Acresce, ainda, a promoção de uma percepção de eficácia pessoal realista, bem como de um auto-conceito mais positivo.

\section{Organização do Programa SABER}

$\mathrm{Na}$ versão actual do programa SABER, todas as sessões foram pensadas para alunos de $7^{\circ}$ ano de escolaridade do Ensino Básico. Esta condicionante decorre de um esforço havido de interligar as actividades do programa aos conteúdos curriculares das matérias estudadas pelos alunos. A duração de cada sessão é de 90 minutos.

Todas as sessões têm a mesma estrutura. Iniciam-se com uma revisão reflexiva das estratégias treinadas na sessão anterior, seguindo-se o preenchimento da agenda para a semana subsequente. De seguida, procede-se à administração, realização, correcção e discussão das actividades de treino das estratégias de aprendizagem e dos processos cognitivos associados. Por fim, procede-se à reflexão sobre as aprendizagens efectuadas na sessão, culminando esta com o preenchimento da ficha de integração, visando a metacognição, consciencialização e uso autónomo e auto-regulado das estratégias aprendidas. Esta ficha de integração tem como base as dimensões da auto-regulação da aprendizagem explicitadas por Zimmerman e Risemberg (1997). Por fim, todas as fichas, registos e trabalhos são guardados numa pasta ou portfolio. Este portfolio tem como intenção constituir-se como um arquivo dinâmico, no qual os alunos podem verificar os seus progressos, as suas dificuldades iniciais ultrapassadas e as que subsistem, bem como as suas avaliações e reflexões (através das fichas de integração) e constituir-se como exemplo/modelo para as actividades quotidianas dos alunos, dada as componentes de avaliação e reflexão associadas (Bernardes \& Miranda, 2003; Mukarugagi, 2002).

Mais objectivamente, são 12 as sessões que compõem o programa SABER, as quais se encontram agrupadas em 4 módulos: planeamento (transversal ao programa); processos básicos de processamento da informação; processos de transformação da informação; e, processos de elaboração da resposta. No primeiro módulo integram-se as primeiras quatro sessões do programa, no segundo módulo incluem-se as sessões 5, 6 e 7; o terceiro módulo abarca as sessões 8, 9 e 10; finalmente, a sessão 11 é abrangida pelo $4^{\mathrm{o}}$ módulo, concluindo o programa uma sessão de síntese. 
A primeira sessão tem como objectivo principal estabelecer a relação e definir a dinâmica interaccional intrínseca às sessões. Deste modo, nesta sessão, após uma actividade de cariz lúdico de quebra-gelo e visando a apresentação, explora-se a linha de base do conhecimento sobre estratégias de estudo. Posteriormente, auscultam-se as expectativas sobre o programa e revela-se a estrutura do mesmo, apresentando o Mirpur, o ser alienígena que acompanhará os alunos ao longo do programa. O Mirpur tem como função lançar a discussão sobre algumas estratégias, bem como servir de modelo de regulação motivacional e metacognitiva. Por fim, procede-se à definição das normas de funcionamento do grupo e faz-se referência à forma de avaliação de cada sessão através da apresentação da ficha de integração e dos objectivos da mesma.

$\mathrm{Na}$ segunda e terceira sessões o programa SABER visa intervir no planeamento e gestão do tempo, através da construção de calendários das actividades trimestrais pessoais de cada aluno e de agendas semanais, bem como através da realização de tarefas com vista à promoção da tomada de consciência da importância da distribuição das actividades pelo tempo, tomando critérios, como por exemplo, a hierarquização de objectivos e o grau de dificuldade e/ou interesse das mesmas. $\mathrm{Na}$ quarta sessão, o objectivo primordial é o planeamento e gestão do espaço e do material de estudo. Nesta sessão é importante salientar a construção da folha de registo das faltas de material, a ser revista ao longo do programa. Este conjunto inicial de sessões visa conduzir os alunos à compreensão de que, previamente a qualquer actividade, nomeadamente, de estudo e de aprendizagem, tem de haver uma fase de planeamento de objectivos e de estratégias. Essa fase repercute-se na gestão do tempo, do espaço e dos materiais de estudo.

Após a planificação, é importante desenvolver estratégias que auxiliem a manter a atenção de modo a realizar de forma eficaz as tarefas de aprendizagem. Garantida a atenção, é possível memorizar os conteúdos. Nas sessões subsequentes são treinadas estratégias que permitem aos alunos a focalização e a manutenção da atenção nos contextos sala de aula e estudo individual, bem como algumas estratégias que lhes permitem memorizar com mais facilidade os conteúdos a aprender. Deste modo, entre a quinta e a sétima sessão, a intervenção tem como alvo os processos básicos de processamento da informação (segundo módulo). Assim, na quinta sessão, trabalha-se a atenção no estudo em casa, começando por uma actividade de promoção da discussão sobre a definição do processo de atenção. Seguidamente, propõe-se aos alunos a realização de uma folha de registo dos factores de distracção e das estratégias usadas para os controlar (a preencher transversalmente ao longo do programa), trabalhando-se a técnica do sublinhado como meio de salientar a informação relevante. $\mathrm{Na}$ sexta sessão, trabalha-se o processo de atenção na sala de aula, através de uma actividade sobre os principais factores de distracção na sala de aula e também, através de outra actividade que exigia o recurso a aulas simuladas, utilizando o projector multimédia. Pretende-se com esta última actividade relevar a importância dos aspectos paralinguísticos da comunicação, que devem servir de sinais para discriminar os conteúdos relevantes dos mais 
anódinos no discurso dos professores durante as aulas. Na sétima sessão, trabalha-se a memorização, encarada como um processo duplo de retenção e de recuperação. Todas as actividades propostas visam a compreensão da necessidade de se criarem pistas (associação de ideias, construção de imagens mentais, associações a lugares de um percurso específico - mnemónica dos lugares), de acordo com o princípio de codificação específica (Tulving \& Thomson, 1973) para garantir a acessibilidade da informação retida aquando da sua recuperação.

Atendendo a que o material a aprender deve ser integrado em estruturas com significado (Moreira \& Buchweitz, 2000) é importante promover, além de estratégias de memorização, estratégias de compreensão do mesmo. Deste modo, nas sessões subsequentes promove-se a internalização de estratégias de transformação da informação, necessárias à compreensão da informação, tomando este processo como uma construção-integração de conhecimentos (Kintsch, 1998). Desta forma, entre a oitava e a décima sessão, a intervenção foca-se em processos de transformação, em particular, na compreensão. Assim, na oitava sessão é trabalhada a técnica $S Q 3 R$ - Survey, question, read, recite, review -, de modo a auxiliar os alunos a desenvolverem estratégias de compreensão de textos narrativos e expositivos. Na nona sessão, são trabalhadas as técnicas de clarificação, envolvendo a consulta quer de dicionários e enciclopédias, quer dos próprios manuais, e as técnicas do resumo e da esquematização. Com o objectivo de preparar os alunos para os anos subsequentes e para a progressiva complexificação dos conteúdos curriculares, faz-se ainda uma abordagem superficial à técnica dos mapas de conceitos. Na décima sessão, visando promover o desenvolvimento de estratégias de compreensão no âmbito de sala de aula e recorrendo a aparelhos multimédia com vista à visualização e audição de aulas simuladas, treina-se a técnica de tomada de notas.

O fim do processamento da informação é produzir uma resposta, uma acção. Deste modo, a décima primeira sessão integra o módulo de elaboração de resposta. Tem como objectivo primordial treinar o pensamento divergente, tomando conteúdos de disciplinas como Português, Educação Visual e Matemática. Tendo em consideração os critérios de avaliação da criatividade explicitados por Guilford (1983) fluência, flexibilidade, originalidade e elaboração-, treinam-se os alunos na internalização destes critérios e do seu uso com o intuito de realizar produções criativas. Por fim, a última sessão consta de uma série de actividades com o objectivo de aplicar todos os conhecimentos e estratégias construídos ao longo das sessões. Com vista a "completar um ciclo" os alunos preenchem novamente a ficha de avaliação da linha de base dos conhecimentos sobre estratégias de aprendizagem e são levados a comparar as respostas com aquelas que haviam dado no início do programa. No final, cada aluno recebe de forma definitiva e num acto simbólico, o seu respectivo portfolio.

Apresentado o programa SABER em termos da sua organização, importa explicitar, por último, a dinâmica das próprias sessões. Todas as actividades são realizadas, primeiro individualmente, promovendo-se de seguida a discussão entre os alunos, com o objectivo de favorecer o conflito sócio-cognitivo. Também se pretendeu desenvolver 
a tomada de perspectiva do outro, solicitando ao grupo que explicasse o raciocínio subjacente à opinião do aluno que se expressava. Além disso, todas as sessões contam com a presença de um ser alienígena (o Mirpur, de Zorax) que tem como objectivo, por um lado, fomentar a discussão sobre as percepções dos alunos relativamente às estratégias e processos envolvidos no estudo, e por outro, servir de modelo não só ao nível do delineamento estratégico das resoluções das tarefas, mas também ao nível do controlo volitivo e emocional, com vista à promoção da auto-regulação da aprendizagem.

\section{Conclusão}

À guisa de conclusão podemos afirmar que o programa SABER pretende constituir-se como uma aplicação prática do modelo do Adaptable Learning (Boekaerts, 1996), visando auxiliar os alunos a avaliarem positivamente as situações de aprendizagem e, consequentemente, a definirem objectivos de mestria. Para tal, a mediatização (Feuerstein, 1980; Fonseca, 2001) e a promoção do conflito sócio-cognitivo (Doise \& Mugny, 1997) aparecem utilizados como as estratégias de treino mais valorizadas como forma de promover a internalização de novas formas de abordar, interpretar e avaliar as situações de realização e de avaliação das tarefas cognitivas e de aprendizagem.

Orientado para o desenvolvimento de um estudo e aprendizagem eficazes através da apropriação de processos cognitivos, o programa assume uma sequencialidade nas funções mentais inerente ao processamento da informação, nomeadamente as fases do planeamento, resolução e avaliação. Todo este treino é enquadrado no objectivo da autonomia funcional do aluno, ou seja, a sua auto-regulação. $\mathrm{O}$ desenvolvimento de um portfolio é uma adenda favorável à promoção da auto-regulação, o mesmo se podendo dizer da Ficha de Integração. Além disso, o programa visa treinar, de forma transversal a todas as sessões, a gestão do tempo (particular dificuldade sentida nos alunos portuguesas desta faixa etária), bem como as crenças motivacionais, em particular a percepção de eficácia pessoal, dada a repercussão destas crenças no desempenho das tarefas e na definição de intenções de aprendizagem (Boekaerts, 1999; Seegers \& Boekaerts, 1993).

Pelas características do programa evidenciadas atrás, a sua aplicação tem algumas implicações. Antes de tudo, o facto do programa se basear no curriculum do $7^{\circ}$ ano de escolaridade do Ensino Básico, impõe que a sua aplicação se restrinja a este ano de escolaridade. Além disso, dado que a sua organização está estruturada em sessões com a duração de 90 minutos, é importante que as escolas assumam esta característica do programa e prevejam salas e horários ajustados.

Por fim, o programa SABER requer a formação específica dos aplicadores. Esta formação deverá ser ao nível teórico, bem como ao nível técnico (conhecer e compreender as actividades e estratégias utilizadas em cada sessão), Acima de tudo, a formação dos aplicadores deverá contemplar o seu desenvolvimento ao nível das competências de interacção e das crenças sobre a modificabilidade cognitiva, para que possam, da forma mais ajustada, dinamizar as sessões de acordo com os objectivos do programa. 


\section{Referências}

Almeida, L. S. (1993). Rentabilizar o ensinoaprendizagem escolar para o sucesso e o treino cognitivo dos alunos. Em L. S. Almeida (Ed.), Capacitar a escola para o sucesso: Orientações para a prática educativa (pp. 59-110). Vila Nova de Gaia: Edipsico.

Ausubel, D. P. (1968). Educational Psychology: A cognitive view. Nova York: Holt, Rinehart and Winston.

Bernardes, C., \& Miranda, F. B. (2003). Portefolio: Uma escola de competências. Porto: Porto Editora.

Boekaerts, M. (1992). The adaptable learning process: Initiating and maintaining behavioural change. Applied Psychology: An International Review, 41, 377-397.

Boekaerts, M. (1996). Coping with stress in childhood and adolescence. Em M. Zeidner \& N. S. Endler (Eds.), Handbook of coping. Theories, research, applications (pp.452-484). Nova York: Wiley.

Boekaerts, M. (1999). Motivated learning: Studying student situation transactional units. European Journal of Psychology of Education, XIV(1), 41-55.

Boekaerts M., \& Niemivirta, M. (2000). Self-regulated learning: Finding a balance between learning goals and ego-protective goals. Em M. Boekaerts, P. R. Pintrich \& M. Zeidner (Eds.), Handbook of self-regulation (pp.417450). San Diego: Academic Press.

Doise, W., \& Mugny, G. (1979). Factores sociologicos y psicosociologicos del desarrollo cognitivo: Una nueva ilustración experimental. Anuário de psicologia, 21, 425.

Doise, W., \& Mugny, G. (1997). Psicologia social $e$ desenvolvimento cognitivo. Colecção Epigénese, desenvolvimento e psicologia. Lisboa: Instituto Piaget.

Feuerstein, R. (1980). Instrumental Enrichment. Baltimore: University Park Press.
Fonseca, V. (2001). Cognição e aprendizagem. Lisboa: Âncora Editora.

González-Pienda, J. A. (2003). Enseñar y aprender significativamente: Un reto para professores y alumnos. Revista Galego-Portuguesa de Psicoloxia e Educación, 10(8), 159-171.

Guilford, J. P. (1983). Creatividad y educación. Buenos Aires: Paidós Educador.

Kintsch, W. (1998). Comprehension: Paradigm for cognition. Cambridge: Cambridge University Press.

Monereo, C., Pozo, J. I., \& Castelló, M. (2001). La enseñanza de estratégias de aprendizaje en el contexto escolar. Em C. Coll, J. Palácios \& A. Marchesi (Eds.), Desarrollo psicológico y educación 2, y psicologia de la educación escolar (pp.235-258). Madrid: Alianza Editorial.

Moreira, M. A., \& Buchweitz, B. (2000). Novas estratégias de ensino e de aprendizagem: Os mapas conceptuais e o Vê epistemológico. (2 $2^{\mathrm{a}}$ ed.). Colecção Aula prática. Lisboa: Plátano Edições Técnicas.

Mukarugagi, L. (2002). Le portfolio, engagemant de l'élève dans l'évaluation de ses apprentissages. Montréal: McGraw-Hill.

Novak J. D., \& Gowin, D. B. (1999). Aprender a aprender ( $2^{\mathrm{a}}$ ed.). Colecção Plátano Universitária. Lisboa: Plátano Edições Técnicas.

Perret-Clermont, A. N. (1995). Desenvolvimento da inteligência e interacção social ( $2^{\mathrm{a}}$ ed.). Colecção Horizontes pedagógicos. Lisboa: Instituto Piaget.

Rosário, P. S. L. (2004). (Des)venturas do TESTAS: Estudar o estudar. Porto: Porto.

Roux, J-P. (2003). Analyse interlocutoire, dynamiques interactives et étude des mécanismes des progrès cognitifs en situation asymétrique de résolution de problèmes. L'Orientation Scolaire et Professionnelle, 32(3), 475-501. 
Seegers, G., \& Boekaerts, M. (1993). Task motivationand mathematics achievement in actual task situations. Learning and Instruction, 3, 133-150.

Trognon, A. (1999). Éléments de l'analyse interlocutoire. Em M. Gilly, J-P. Roux \& A. Trognon (Eds.), Apprendre dans l'interaction.Analyse des médiations sémiotiques (pp.69-94). Aix-en-Provence et Nancy : Publications de l’Université de Provence et P.U.N..

Tulving, E., \& Thomson, D. M. (1973). Encoding specificity and retrieval processes in episodic memory. Psychological Review, 80, 352-373.

Veiga Simão, A. M. (2002). Aprendizagem estratégica: Uma aposta na auto-regulação. Colecção desenvolvimento curricular. Lisboa: I.I.E. - Ministério da Educação.

Veiga Simão, A. M. (2004). O conhecimento estratégico e a auto-regulação da aprendizagem: Implicações em contexto escolar. Em A. Lopes da Silva, A. M. Duarte, I. Sá \& A. M. Veiga Simão (Eds.), Aprendizagem autoregulada pelo estudante: Perspectivas psicológicas $e$ educacionais (pp.77-95). Porto: Porto.

Vygotsky, L. S. (1978). Mind in society: the development of higher psychological processes. Cambridge: Harvard University Press

Vygotsky, L. S. (2003). A formação social da mente (6 ed.). São Paulo: Martins Fontes.

Zimmerman, B. J. (2000). Attaining self-regulation: A social cognitive perspective. Em M. Boekaerts, P. R. Pintrich \& M. Zeidner (Eds.), Handbook of selfregulation (pp.13-39). San Diego: Academic Press.

Zimmerman, B. J., \& Risemberg, R. (1997). Becoming a self-regulated writer: A social cognitive perspective. Contemporary Educational Psychology, 22, 73-101. 